\title{
Systematic Review of Ocular Involvement of SARS-CoV-2 in Coronavirus Disease 2019
}

\author{
Kai Xiong Cheong ${ }^{1}$ (D) \\ Accepted: 17 September 2020 / Published online: 26 September 2020 \\ (C) Springer Science+Business Media, LLC, part of Springer Nature 2020
}

\begin{abstract}
Purpose of Review Studies have reported ocular involvement in the coronavirus disease 2019 (COVID-19), with SARS-CoV-2 having been detected in ocular swab samples. This has implicated the eye as a portal of transmission. The aim of this systemic review is to summarise and discuss the current literature regarding ocular involvement of SARS-CoV-2 in COVID-19.

Recent Findings In this systematic review, the prevalence of ocular symptoms and signs was low (from 0 to $31.58 \%$ ) and conjunctivitis was a relatively rare occurrence. The rate of detection of SARS-CoV-2 in the ocular swab samples was low as well and this ranged from 0 to $11.11 \%$. The development of ocular symptoms and signs was not always accompanied by the detection of SARS-CoV-2 in the ocular swab samples. The opposite was described as well. This may reflect issues related to the characteristics of SARS-CoV-2 and of the study design. Nonetheless, the nature of research in a pandemic is that conclusions can change as more information is obtained.

Summary Whilst the eye is unlikely to be a main transmission route, we need to consider the possibilities of conjunctivitis as a presenting complaint and of the eye playing a role in the transmission of SARS-CoV-2. Furthermore, we need to take the appropriate precautions in our practice. Further studies are needed to evaluate the viral tropism of SARS-CoV-2 and its role in the eyes.
\end{abstract}

Keywords Coronavirus disease $2019 \cdot$ SARS-CoV-2 $~$ Conjunctiva $\cdot$ Tears $\cdot$ Transmission $\cdot$ Reverse transcription polymerase chain reaction $\cdot$ Viral culture

\section{Introduction}

The SARS-CoV-2, which is a novel betacoronavirus, has caused a severe acute respiratory syndrome termed as the coronavirus disease 2019 (COVID-19) since December 2019. This year, globalisation and modern travel have facilitated the transmission of this novel virus worldwide within a few months of its origin. Consequently, SARS-CoV-2 has imposed a tremendous burden on the healthcare infrastructure and economy. The World Health Organization subsequently declared the outbreak a pandemic on 11 March 2020. The cumulative disease burden and mortality caused by SARS-CoV-2 have surpassed those of both SARS$\mathrm{CoV}$ and MERS-CoV, which caused the Severe Acute Respiratory Syndrome (SARS) in 2003 and the Middle East

Kai Xiong Cheong

cheong.kai.xiong@singhealth.com.sg

1 Singapore Eye Research Institute, Singapore National Eye Centre, 11 Third Hospital Avenue, Singapore 168751, Singapore
Respiratory Syndrome (MERS) in 2012 respectively, with approximately 13.62 million cases and 585,727 deaths globally [1].

Like other respiratory viruses, SARS-CoV-2 is purported to be transmitted via respiratory and contact routes [2]. Faecal-oral transmission has been reported as well [3]. In addition, there is a growing body of evidence from studies that have reported ocular involvement in COVID-19, with reports of detection of SARSCoV-2 in ocular swab samples [4-20]. This has implicated the eye as an additional portal of transmission.

Ocular transmission of COVID-19 was not reported initially. It began with anecdotal reports of a red eye preceding the onset of respiratory symptoms and pneumonia [21, 22]. One prominent example is Dr. Guangfa Wang, who is a respiratory medicine specialist from the Beijing's Peking University First Hospital. He was part of the panel from the National Health Commission of the People's Republic of China which investigated the pneumonia outbreak at Wuhan. Whilst he wore a N95 mask, his eyes were not protected. It was speculated that ocular exposure allowed SARS-CoV-2 to infect the body. Subsequently, a report in the Lancet highlighted the issue that ocular surfaces may be a potential portal through which 
SARS-CoV-2 can infect the body [22]. There are also reports in which ophthalmologists were found to be infected through routine work as well [21].

The implications of ocular transmission of SARS-CoV-2 are the risks of contracting COVID-19 by healthcare workers and of inadvertent transmission to patients. Healthcare workers work in close proximities to patients. The working distances of the slit lamp and the indirect binocular ophthalmoscopes, for example, bring to mind the potential risks of disease spread between ophthalmologists and patients due to infected respiratory droplets and contact.

To date, there are many studies which have described ocular involvement in COVID-19 and have examined the role of the eye in transmitting SARS-CoV-2 [4-20]. To this end, different research teams have performed reverse transcription polymerase chain reaction (RT-PCR) on ocular samples of tears and conjunctival secretions, and viral cultures. What has been reported is that COVID-19 can be accompanied by conjunctivitis with its attendant symptoms of conjunctival infection, epiphora, itch, discharge, etc., but this remains a relatively rare occurrence compared with the classic respiratory symptoms [4-20]. It is questionable whether the eye is truly a portal of transmission. What is also unknown is the relative significance of this portal compared with other conventional means like respiratory droplets. Whilst some studies have reported the presence of the virus in ocular samples in eyes without conjunctivitis, others have reported the opposite [4-20]. It is also difficult to ascertain whether the presence of the virus indicates its replication within conjunctival cells or viral shedding due to viraemia. Part of this problem can be attributed to the paucity of studies which demonstrate cytopathogenic effects in host cells as well.

The aim of this systemic review is therefore to summarise and discuss the current literature regarding ocular involvement of SARS-CoV-2 in COVID-19.

\section{Methods}

A comprehensive and systematic MEDLINE search of peerreviewed articles using PubMed was performed, with

\section{Table 1 Search terms}

\begin{tabular}{lll}
\hline No. & Search terms & Results \\
\hline 1 & Ophthalmology AND (COVID OR Coronavirus) & 273 \\
2 & Ocular AND (COVID OR Coronavirus) & 135 \\
3 & Ophthalmic AND (COVID OR Coronavirus) & 376 \\
4 & Eye AND (COVID OR Coronavirus) & 338 \\
5 & Conjunctivitis AND (COVID OR Coronavirus) & 72 \\
6 & Conjunctiva AND (COVID OR Coronavirus) & 34 \\
7 & Tears AND (COVID OR Coronavirus) & 71 \\
\hline
\end{tabular}

coverage up to 10 July 2020 . The search strategy with the search terms and Boolean operators is described in Table 1. Observational studies which both described ocular involvement among patients with COVID-19 and attempted to detect SARS-CoV-2 in ocular samples via RT-PCR and/or viral cultures were included. Case series, case reports, and correspondences were also included. The listed references in the selected articles were further examined for additional relevant articles. No unpublished and non-peer-reviewed studies were included in this systematic review. All searches were limited to the English language. A total of 54 studies were reviewed, of which 17 papers met the inclusion criteria and were consequently reviewed (see Fig. 1 for the Preferred Reporting Items for Systematic Reviews and Meta-Analyses (PRISMA) flowchart).

\section{Summary of Study Findings}

The 17 studies are summarised in Table 2 [4-20].

The largest study in this review was by Zhou et al. [4]. This was a cross-sectional study of 121 patients. Of these 121 patients, eight patients (6.61\%) experienced ocular symptoms and signs, including conjunctival injection, itching, epiphora, discharge, and foreign body sensation. Furthermore, three patients $(2.48 \%)$ out of 121 patients had SARS-CoV-2 ribonucleic acid (RNA) detected in the conjunctival swabs. Of these three patients, only one had ocular symptoms and signs, and the remaining two had no ocular symptoms and signs. Zhou et al. also commented that the disease duration was not correlated with either the presence of ocular symptoms and signs or the presence of SARS-CoV-2 in the conjunctival swab samples [4].

Karimi et al. performed a prospective case series which involved 43 patients [7], of whom two patients (4.65\%) experienced ocular symptoms and signs. Of these two patients, one patient had symptoms of conjunctival congestion and mucous discharge, and the other patient experienced foreign body sensation. SARS-CoV-2 RNA was detected in the conjunctival and tear samples of three patients $(6.98 \%)$ out of 43 patients. Only one out of these three patients had ocular symptoms and signs of conjunctival congestion and mucous discharge, and the remaining two did not experience ocular symptoms and signs. In all three cases, SARS-CoV-2 RNA was detected both in the conjunctival and tear samples and in the nasopharyngeal samples. In contrast, there was no conjunctival or tear positive cases among the nasopharyngeal negative cases. Karimi et al. suggested that this was because the viral load in the nasopharynx is probably higher than that in the tears [7].

$\mathrm{Wu}$ et al. examined 38 patients in another retrospective case series [8]. Among these 38 patients, two patients $(5.26 \%)$ yielded positive findings for SARS-CoV-2 in both conjunctival and nasopharyngeal specimens. A total of 12 
Fig. 1 PRISMA flowchart

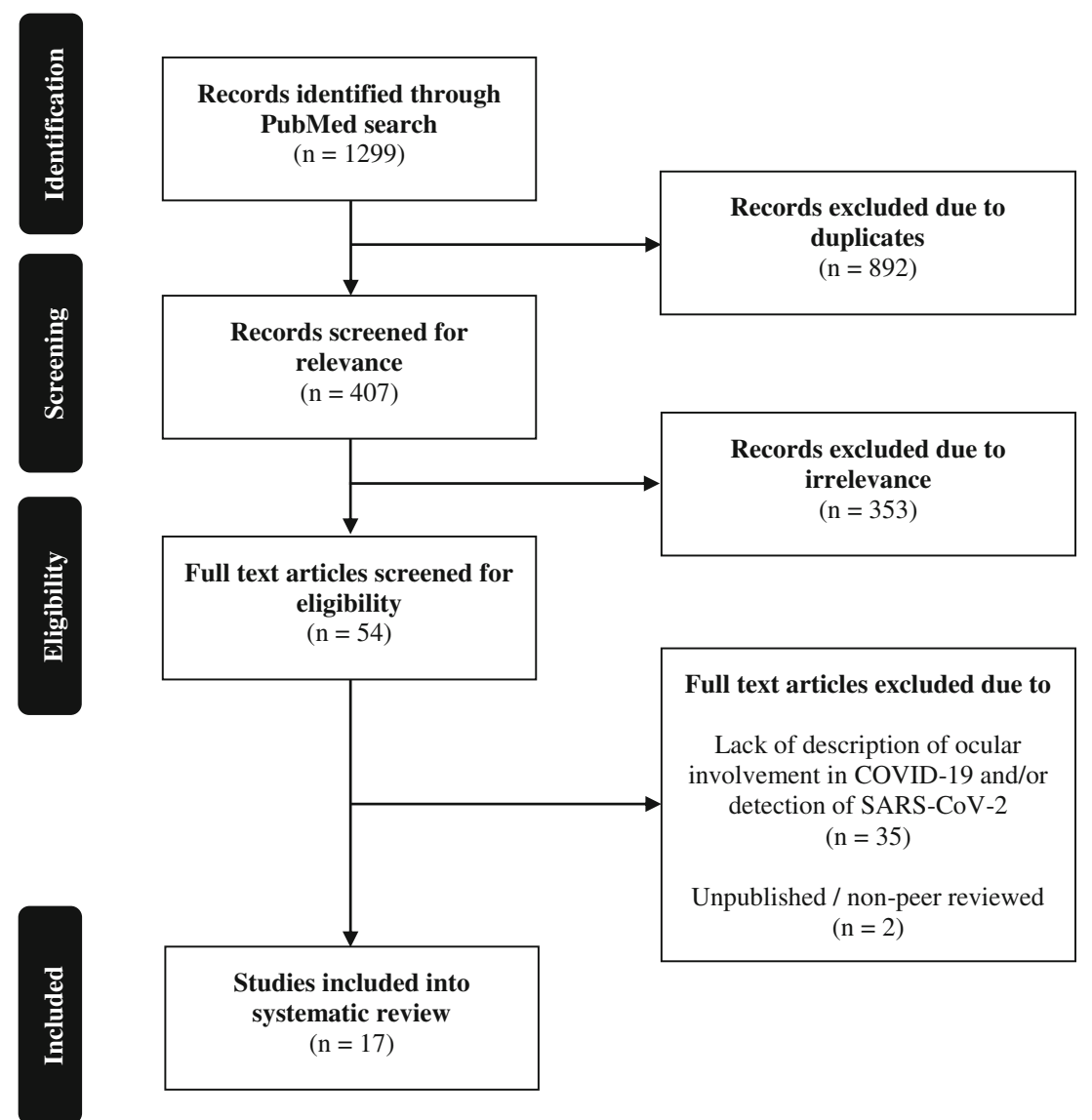

out of 38 patients $(31.58 \%)$ experienced ocular symptoms and signs consistent with conjunctivitis, including conjunctival injection, chemosis, epiphora, and secretion. Among these 12 patients, there were four, two, and six patients whose conditions were assessed to be moderate, severe, and critical, respectively. The conditions of these 12 patients were graded according to the National Guidelines on Prevention and Control of the Novel Coronavirus Pneumonia (PC-NCP) [8]. It was also reported in a univariate analysis that patients with more severe pneumonia were more likely to experience ocular symptoms and signs, with higher levels of white blood cells, neutrophils, procalcitonin, C-reactive protein, and lactate dehydrogenase compared with patients without ocular symptoms and signs [8].

The ocular manifestations and prevalence of SARS-CoV-2 in 27 children were evaluated by Valente et al. in a prospective case series [12]. Out of 27 patients, ocular symptoms and signs consistent with mild viral conjunctivitis including conjunctival injection and secretion were observed in four patients $(14.81 \%)$. SARS-CoV-2 RNA was detected in three $(11.11 \%)$ out of 27 patients. Of these three patients, only one had ocular symptoms and signs. The conjunctival swabs of the two asymptomatic patients and of the symptomatic patient became negative at the second swab (3 days) and at the third swab (6 days) respectively, whilst the nasopharyngeal swabs remained positive. The authors went on to suggest that the ocular manifestations of COVID-19 appeared to take a milder clinical course in the paediatric population compared with adults [12].

In three different studies, Zhang et al. [5], Xia et al. [11], and Guemes-Villahoz et al. [14] reported that SARS-CoV-2 was detected in the ocular samples from patients with ocular symptoms and signs. In a retrospective case series of 72 patients, Zhang et al. described that two patients $(2.78 \%)$ experienced ocular symptoms and signs of conjunctival congestion and watery discharge. SARS-CoV-2 RNA was detected in the conjunctival swabs of only one patient (1.39\%) out of 72 patients, and this patient was one of the two patients who had ocular symptoms and signs [5]. Similarly, in a prospective case series of 30 patients, Xia et al. observed that SARS-CoV-2 was detected in the conjunctival swab and tear samples from one patient $(3.33 \%)$. This patient was also the only one with conjunctivitis. Correspondingly, no viral RNA was detected in samples from patients without conjunctivitis [11]. However, SARS-CoV-2 was not successfully isolated and cultured. Guemes-Villahoz et al. performed a cross-sectional study of 14 COVID-19 patients who were diagnosed with conjunctivitis and on whom conjunctival swabs were performed [14]. 


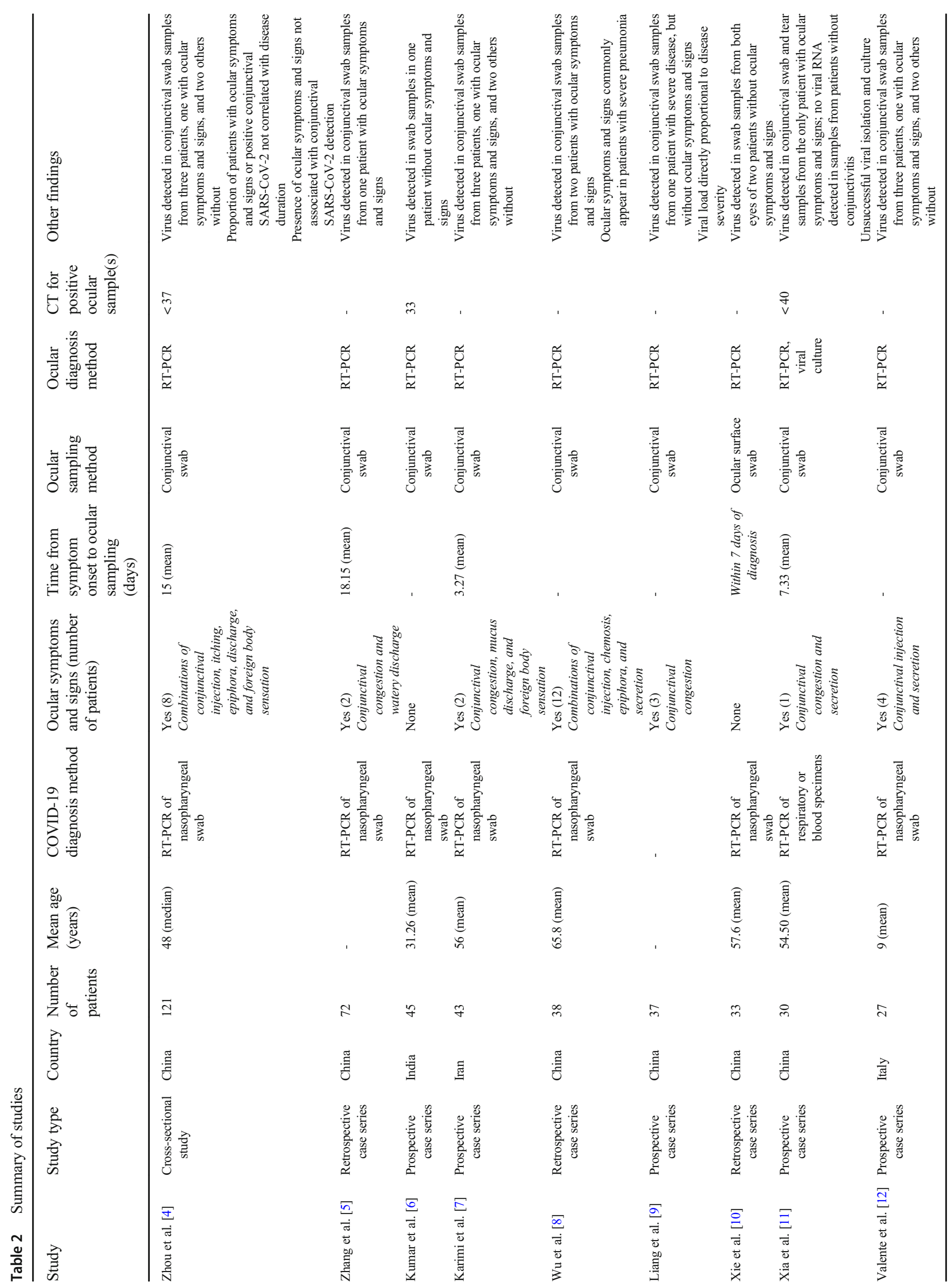




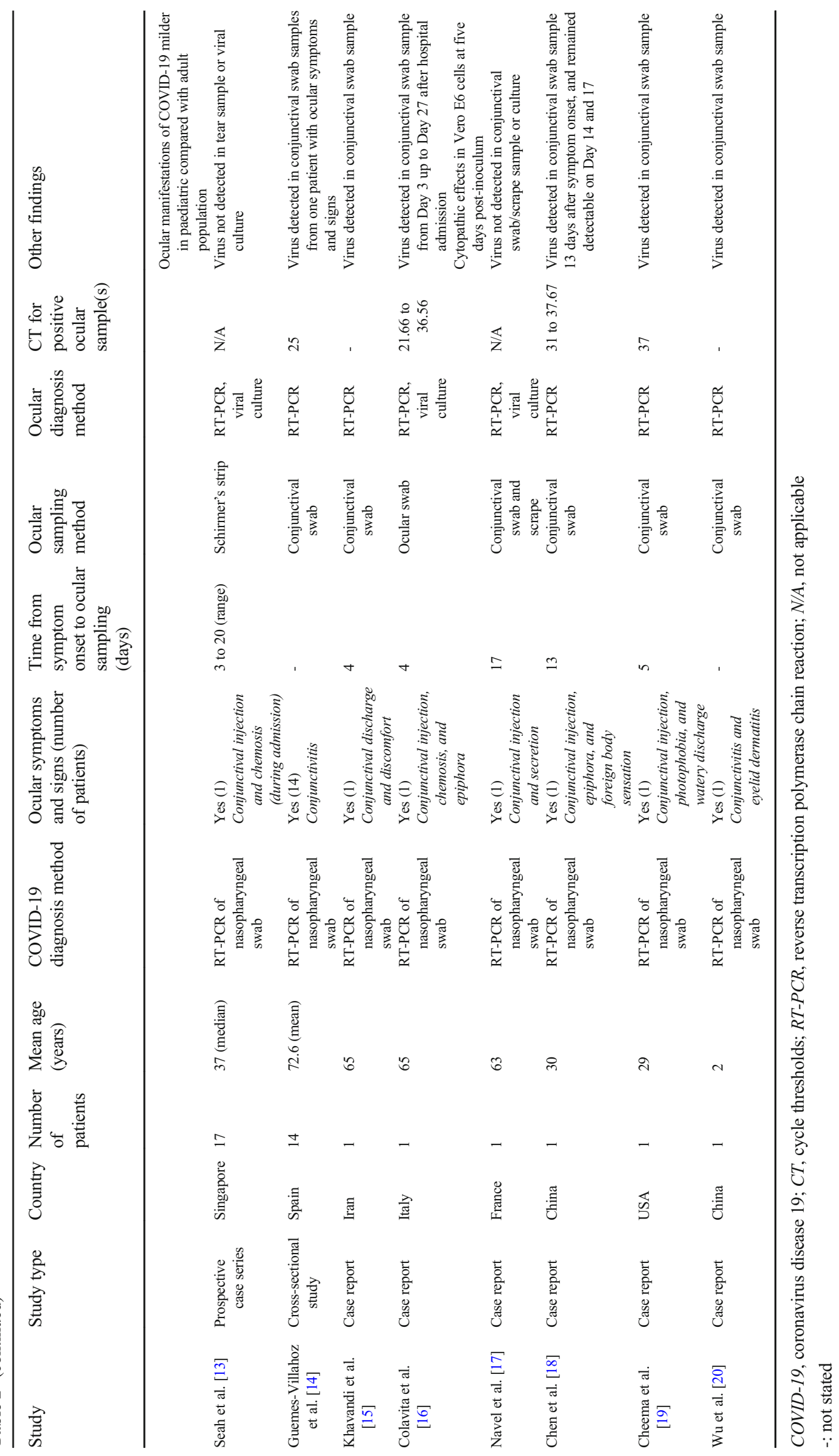


Among these 14 patients, SARS-CoV-2 RNA was detected in conjunctival swab of only one patient $(7.14 \%)$.

In contrast, other studies have reported the detection of SARS-CoV-2 in ocular samples from patients who did not experience ocular symptoms and signs $[5,9,10]$. In a prospective case series, Kumar et al. described in a group of 45 patients that SARS-CoV-2 was detected in conjunctival swab samples from one patient $(2.22 \%)$ [5]. None of the patients in the series had ocular symptoms and signs. In another prospective case series, Liang et al. described in a group of 37 patients that SARS-CoV-2 was detected in conjunctival swab samples from one patient $(2.70 \%)$ with severe disease. This patient did not have conjunctivitis [9]. In contrast, three other patients $(8.11 \%)$ out of 37 patients had ocular symptoms and signs, including conjunctival congestion, but did not have positive conjunctival swabs. Liang et al. also commented that the viral load was directly proportional to disease severity [9]. Similar to that reported by Liang et al., Xie et al. reported in a retrospective case series that SARS-CoV-2 was detected in conjunctival swab samples from both eyes of two patients $(6.06 \%)$ in a population of 33 patients. Both of these patients did not have ocular symptoms and signs [10].

Seah et al., in a prospective case series of 17 patients, reported that SARS-CoV-2 could not be detected in RT-PCR of tear samples. Cytopathogenic effects were not observed in viral cultures, even when the nasopharyngeal swabs continued to test positive [13]. The authors concluded that there was a low risk of transmission of SARS-CoV-2 from the tears [13]. Notably, the tear samples were obtained between day 3 and day 20 from the onset of (respiratory) symptoms to give a wider coverage of different phases of the infection course. It is important to note that no patients presented with ocular symptoms and signs, although one patient (5.88\%) out of 17 patients developed conjunctival injection and chemosis during admission [13].

The remaining are case reports by Khavandi et al. [15], Colavita et al. [16], Navel et al. [17], Chen et al. [18], Cheema et al. [19], and $\mathrm{Wu}$ et al. [20]. These case reports are of patients of various ages, including a child. Whilst the reports generally described the development of ocular symptoms and signs that are suggestive of conjunctivitis, Navel et al. [17] and Cheema et al. [19] reported the development of pseudomembranous haemorrhagic conjunctivitis and keratoconjunctivitis, respectively. These manifestations were reported associations and were not tested for causality.

Colavita et al. [16] and Chen et al. [18] also described the evolution of the cycle threshold (CT) values from the RT-PCR analyses of the ocular swab samples. In the study by Colavita et al., ocular swab samples were taken from one patient from day 3 to day 30 from the onset of ocular and respiratory symptoms [16]. SARS-CoV-2 was detected in the ocular swab samples until day 21 (CT range: 21.66 to 36.56 ), became undetectable from day 22 to day $26(\mathrm{CT}>45)$, was detected again on day 27 (CT: 36.25), and remained undetectable from day 28 to day $30(\mathrm{CT}>45)$. This was thought to suggest sustained conjunctival replication. The conjunctivitis also improved by day 15 and resolved at day 20 . This was also accompanied by the observation of cytopathogenic effects at 5 days post-inoculation of ocular sample in Vero E6 cells. The viral replication was confirmed by RT-PCR of RNA that was purified from spent cell growth medium. In the study by Chen et al., RT-PCR demonstrated the presence of SARSCoV-2 in the conjunctival swab sample 13 days after onset of symptoms (CT: 31$)$. Conjunctival swab samples remained positive for SARS-CoV-2 on day 14 (CT: 31) and on day 17 (CT: 37.67) and became negative on day 19 (CT > 40) [18].

\section{Discussion}

\section{Findings}

In an attempt to elucidate the ocular impact and implications of the SARS-CoV-2 infection, I have presented a systematic review of 17 papers on the ocular involvement and detection of this novel virus in patients. One of the major conclusions which one can make from this systematic review is that the prevalence of ocular symptoms and signs is low and that conjunctivitis is a relatively rare occurrence. In this review, this prevalence ranges from 0 to $31.58 \%$ [4-20]. This is in keeping with a previous large study from China which reported that nine patients $(0.82 \%)$ out of 1099 laboratory-confirmed COVID-19 patients experienced conjunctival congestion [23]. These patients had reported having experienced various combinations of the following symptoms and signs, including conjunctival injection, congestion, chemosis, itching, epiphora, discharge, and foreign body sensation. In this systematic review, apart from conjunctivitis, there were also descriptions of the development of pseudomembranous haemorrhagic conjunctivitis [13] and keratoconjunctivitis [15] in the patients. It was also suggested that ocular symptoms and signs commonly appear in patients with severe pneumonia [6]. The timing of the onset of ocular manifestations varied among the studies.

The rate of detection of SARS-CoV-2 in the ocular swab samples was low as well, ranging from 0 to $11.11 \%$ with a range of CT from 21.66 to less than 40 [4-20]. This demonstrates a low prevalence of the SARS-CoV-2 in ocular secretions and tears, and that ocular samples may contain SARSCoV-2 RNA in the absence of ocular manifestations. In the studies that took serial samples, SARS-CoV-2 was reported to remain detectable up to 27 days after the onset of ocular and respiratory symptoms $[16,18]$. One case report also observed cytopathogenic effects of SARS-CoV-2 at 5 days postinoculation of ocular sample in Vero E6 cells [16]. The viral 
replication was confirmed by RT-PCR on RNA that was purified from spent cell growth medium [16].

In the studies in this systematic review, the development of ocular symptoms and signs was not always accompanied by the detection of SARS-CoV-2 in the ocular swab samples. The opposite was described as well. This may reflect a myriad of issues related to the characteristics of SARS-CoV-2 and of the study design which need to be addressed, or at least acknowledged.

Whilst these observations do establish an association between SARS-CoV-2 and the eye, it is not as clear whether SARS-CoV-2 is necessarily associated with conjunctivitis and how the eye plays a causal role in transmitting the virus. The presence of SARS-CoV-2 RNA in the ocular swab samples does not necessarily mean that SARS-CoV-2 can enter the conjunctival epithelial cells and replicate. There are other possibilities, including reaching the conjunctiva via infected respiratory droplets, or via exudation into the ocular samples due to viraemia during the acute phase [24-28].

\section{Study Design}

There are a few possibilities regarding why the results from the studies in this systematic review differed from one another. They can be categorised into study design and patient factors.

Very few studies have provided information regarding the exact protocols and performance standards of their diagnostic test kits and information regarding the positive and negative controls for the RT-PCR and viral culture [28]. Different laboratories could have employed different assays with unique gene target sequences. There could also be issues related to the sensitivity and specificity of RT-PCR [29, 30].

The technique of sampling by trained personnel is also crucial to the accuracy of the results [28]. If not, there may not be sufficient RNA in the ocular samples which can be detected by RT-PCR.

Contamination of ocular samples is another possibility as well [24-28]. SARS-CoV-2 may be present in ocular samples due to transfer of virus onto the ocular surface via infected respiratory droplets, direct contact with contaminated hands, migration of infected secretions from the respiratory tract through the nasolacrimal duct, or secretion from lacrimal glands [24-28].

Not all the studies have described the exact timings of the ocular sample collection. Among the prospective studies, a few authors have reported the timings of ocular sample collection relative to the onset of ocular symptoms and signs. For example, Xia et al. reported that the first collection of tear and conjunctival secretions was performed at a mean of 7.33 days after the onset of ocular symptoms and signs [11]. Seah et al. also described that out of 64 samples, 12, 28, and 24 samples were taken from the first, second, and third weeks from the initial onset of ocular symptoms, respectively [13]. In contrast, many of the remaining studies did not describe the timings of the ocular sample collection.

In addition, these timings are dependent on patient recollection that is inherently subjective. Many of the studies relied on a single sampling time point. Therefore, the elapsed time from the onset of symptoms to the sampling time would have differed among these studies [10, 24]. A relative lag in sampling time in some studies would have caused the viral shedding and viraemia window to be missed and would have consequently resulted in false negative results $[4,10,11,24]$. The treatment durations before ocular sampling was performed also varied, and therefore, treatment effects could have confounded the study results [4].

All these preceding considerations would have necessitated the ocular sampling to be repeated and separated by time. Therefore, the ocular sampling at only one point in time for many of the studies is potentially problematic. Lastly, in many of these studies, understandably, the size of the study populations was relatively small.

\section{Premise for and Mechanisms of Transmission}

The detection of SARS-CoV-2 RNA does not necessarily mean that the virus is present. Shedding of SARS-CoV-2 RNA does not necessarily represent infectivity. Arguments for the transmission of SARS-CoV-2 through the conjunctiva should be predicated upon the demonstration of the replicative capacity of SARS-CoV-2 and the identification of viral particles and cytopathogenic effects in conjunctival epithelial cells $[13,16,24]$. To this end, there are considerations related to the structural properties of the conjunctiva and of SARS-CoV-2.

Wan et al. reported that the sequence of the receptorbinding domain of SARS-CoV-2, including its receptorbinding motif that directly contacts the angiotensinconverting enzyme 2 (ACE2), is similar to that of the SARS-CoV. This strongly suggests that the virus uses ACE2 as its receptor [31]. Previous studies have established that the SARS-CoV spike protein can interact with the human ACE2 protein and infect human respiratory epithelial cells [32].

Correspondingly, it is unclear whether conjunctival epithelia express ACE2 or potential auxiliary proteins and coreceptors [33, 34]. Whilst Lange et al. reported that there was no evidence for a significant expression of ACE2 and its auxiliary mediators for cell entry in conjunctival samples, making conjunctival infection with SARS-CoV-2 via these mediators unlikely [35], Sun et al. reported that ACE2 is expressed in human cornea and conjunctival tissues (conjunctival epithelial cells, conjunctival fibroblast, and corneal epithelial cells) [36].

A recently conducted study demonstrated the capacity of SARS-CoV-2 to infect and replicate in conjunctiva 
epithelium, which may support the findings of the studies in this systematic review [37]. Hui et al. concluded that the conjunctival epithelium appeared to be a potential portal of infection for SARS-CoV-2 [37]. In this study, SARS-CoV-2 from a confirmed COVID-19 patient was isolated. The tropism and replication competence of SARS-CoV-2 were subsequently compared with those of SARS-CoV, MERS-CoV, and the 2009 pandemic influenza H1N1 (H1N1pdm) in ex vivo cultures of human bronchus, lung, colorectal adenocarcinoma, and human conjunctiva. Notably, SARS-CoV-2 replication was demonstrated to be greater than that of SARS-CoV in the conjunctiva [37].

Nonetheless, whilst the eye may play a role in the transmission of SARS-CoV-2, it is unlikely to be a main transmission route [24-28]. This is in consideration of the low detection rates of SARS-CoV-2 in the ocular swab samples and of the high CT values that were reported. Disregarding technical issues related to the study design and diagnostic capabilities, this in turn can be a result of the following: (1) low viral load in ocular tissues; (2) low expression of ACE2 and its auxiliary mediators in the ocular cell membranes; (3) lower affinity of the receptor-binding motif sequence for the conjunctiva compared with the lungs; (4) presence of antimicrobial agents in the tears including immunoglobulin A and lactoferrin; and (5) cleansing and dilution effect of tears on the ocular surface.

As mentioned, the other possible mechanisms leading to the presence of SARS-CoV-2 in ocular swab samples may be from the direct inoculation of the virus on the conjunctiva. This may be further compounded by a degraded ocular surface in the anterior segment due to conjunctivitis, hand-eye contact, and eye rubbing, further allowing access of SARS-CoV-2 $[26,38]$. Other theories which were proposed include the migration of infected secretions from the respiratory tract through the nasolacrimal duct, and even haematogenous infection of the lacrimal gland [24-28, 33]. It was also suggested that transmission of SARS-CoV-2 through tears regardless of the phase of infection is likely to be low [13].

\section{Comparison with Other Viruses}

It is worthwhile noting that in contrast to other respiratory disease viruses like the adenovirus and influenza virus which can cause conjunctivitis or keratoconjunctivitis, coronaviruses are rarely associated with conjunctivitis [34, 39]. There are notable exceptions, one of which is the HcoV-NL63. This virus was first isolated from a 7-month-old child who developed conjunctivitis and bronchiolitis, before being identified in seven other patients [40]. Another retrospective study of 18 patients who were infected with HcoV-NL63 reported that three patients $(16.67 \%)$ developed conjunctivitis [41]. Hitherto to COVID-19, SARS and MERS were not commonly associated with ocular involvement. However, a study using RT-PCR had demonstrated the presence of SARS-
$\mathrm{CoV}$ in tear samples that were collected from 36 patients [42]. High viral loads of MERS-CoV had been reportedly detected in conjunctival swabs of camels as well [43].

\section{Implications}

The significance of this discussion lies in the public health implications. Ophthalmologists routinely examine patients at close proximities. There is a potential for two-way transmission of SARS-CoV-2 between the ophthalmologist and the patients via infected respiratory droplets or contact [44]. Precautionary measures when examining patients with the use of face masks, or even protective goggles and face shields, may be recommended. Instruments such as the air-puff tonometer can cause aerosolisation in theory and pose a risk $[44,45]$. There are also contact risks with the use of slit lamp or binocular indirect ophthalmoscope lenses, contact lenses for laser procedures, trial frames, occluders, and pinhole devices [44]. Currently, mitigation strategies and subspecialty guidelines are being developed [44]. These include, among others, the reduction of patient visits, cancellation of nonessential procedures, training in donning and doffing of personal protective equipment, equipment disinfection protocols, and droplet and fomite precautions [44].

\section{Conclusion}

This systematic review has summarised and discussed the current literature regarding the ocular involvement of SARSCoV-2 in COVID-19. Currently, COVID-19 is posing a threat globally to our health and livelihoods. The prevalence of conjunctivitis and the detection of SARS-CoV-2 in ocular samples so far are low and inconsistent to some extent. However, the nature of research in a pandemic is that conclusions can change as more information is obtained. Whilst the eye is unlikely to be a main transmission route, we need to consider the possibilities of conjunctivitis as a presenting complaint and of the eye playing a role in transmission. We also need to take the appropriate precautions in our practice. Further larger and more comprehensive studies are needed to evaluate the role and viral tropism of SARS-CoV-2 in the eyes.

Author's Contributions Kai Xiong Cheong conceived the idea for this systematic review, performed the literature review and data analysis, and wrote the manuscript.

Data Availability Not applicable

\section{Compliance with Ethical Standards}

Conflict of Interest The author declares that he has no conflict of interest. 
Human and Animal Rights and Informed Consent This article does not contain any studies with human participants or animals performed by any of the authors.

Ethics Approval Not applicable

Consent to Participate Not applicable

Consent for Publication Not applicable

Informed Consent Not applicable

Disclaimer The opinions expressed in this paper are those of the author and do not reflect the official policies, views, or positions of the Singapore Eye Research Institute.

Code Availability Not applicable

\section{References}

1. World Health Organization. Situation reports July 2020. In: Coronavirus disease (COVID-2019) situation reports. World Health Organization. 2020. https://www.who.int/emergencies/ diseases/novel-coronavirus-2019/situation-reports. Accessed 17 Jul 2020.

2. Amirian ES. Potential fecal transmission of SARS-CoV-2: current evidence and implications for public health. Int J Infect Dis. 2020;95:363-70.

3. Holshue ML, DeBolt C, Lindquist S. First case of 2019 novel coronavirus in the United States. N Engl J Med. 2020;382:929-36.

4. Zhou Y, Duan C, Zeng Y, Tong Y, Nie Y, Yang Y, et al. Ocular findings and proportion with conjunctival SARS-COV-2 in COVID-19 patients. Ophthalmology. 2020;127:982-3.

5. Zhang X, Chen X, Chen L, Deng C, Zou X, Liu W, et al. The evidence of SARS-CoV-2 infection on ocular surface. Ocul Surf. 2020;18:360-2.

6. Kumar K, Prakash AA, Gangasagara SB, Rathod SBL, Ravi K, Rangaiah A, et al. Presence of viral RNA of SARS-CoV-2 in conjunctival swab specimens of COVID-19 patients. Indian J Ophthalmol. 2020;68:1015-7.

7. Karimi S, Arabi A, Shahraki T, Safi S. Detection of severe acute respiratory syndrome Coronavirus-2 in the tears of patients with coronavirus disease 2019. Eye (Lond). 2020;34:1220-3.

8. Wu P, Duan F, Luo C, Liu Q, Qu X, Liang L, et al. Characteristics of ocular findings of patients with coronavirus disease 2019 (COVID-19) in Hubei Province, China. JAMA Ophthalmol. 2020;138:575-8.

9. Liang $\mathrm{L}, \mathrm{Wu}$ P. There may be virus in conjunctival secretion of patients with COVID-19. Acta Ophthalmol. 2020;98:223.

10. Xie HT, Jiang SY, Xu KK, et al. SARS-CoV-2 in the ocular surface of COVID-19 patients. Eye Vis (Lond). 2020;7:23.

11. Xia J, Tong J, Liu M, Shen Y, Guo D. Evaluation of coronavirus in tears and conjunctival secretions of patients with SARS-CoV-2 infection. J Med Virol. 2020;92:589-94.

12. Valente P, Iarossi G, Federici M, et al. Ocular manifestations and viral shedding in tears of pediatric patients with coronavirus disease 2019: a preliminary report. J AAPOS. 2020;S1091-8531(20): 30115-4.

13. Seah IYJ, Anderson DE, Kang AEZ, Wang L, Rao P, Young BE, et al. Assessing viral shedding and infectivity of tears in coronavirus disease 2019 (COVID-19) patients. Ophthalmology. 2020;127: 977-9.
14. Güemes-Villahoz N, Burgos-Blasco B, Arribi-Vilela A, et al. SARS-CoV-2 RNA detection in tears and conjunctival secretions of COVID-19 patients with conjunctivitis. J Inf Secur. 2020;81: 452-82.

15. Khavandi S, Tabibzadeh E, Naderan M, Shoar S. Corona virus disease-19 (COVID-19) presenting as conjunctivitis: atypically high-risk during a pandemic. Cont Lens Anterior Eye. 2020;43: 211-2.

16. Colavita F, Lapa D, Carletti F, Lalle E, Bordi L, Marsella P, et al. SARS-CoV-2 isolation from ocular secretions of a patient with COVID-19 in Italy with prolonged viral RNA detection. Ann Intern Med. 2020;173:242-3.

17. Navel V, Chiambaretta F, Dutheil F. Haemorrhagic conjunctivitis with pseudomembranous related to SARS-CoV-2. Am J Ophthalmol Case Rep. 2020;19:100735.

18. Chen L, Liu M, Zhang Z, Qiao K, Huang T, Chen M, et al. Ocular manifestations of a hospitalised patient with confirmed 2019 novel coronavirus disease. Br J Ophthalmol. 2020;104:748-51.

19. Cheema M, Aghazadeh H, Nazarali S, Ting A, Hodges J, McFarlane A, et al. Keratoconjunctivitis as the initial medical presentation of the novel coronavirus disease 2019 (COVID-19). Can J Ophthalmol. 2020;55:e125-9.

20. Wu P, Liang L, Chen C, Nie SQ. A child confirmed COVID-19 with only symptoms of conjunctivitis and eyelid dermatitis. Graefes Arch Clin Exp Ophthalmol. 2020;258:1565-6.

21. Yu AY, Tu R, Shao X, et al. A comprehensive Chinese experience against SARS-CoV-2 in ophthalmology. Eye Vis (Lond). 2020;7: 19.

22. Lu CW, Liu XF, Jia ZF. 2019-nCoV transmission through the ocular surface must not be ignored. Lancet. 2020;395:e39.

23. Guan WJ, Ni ZY, Hu Y, Liang WH, Ou CQ, He JX, et al. Clinical characteristics of coronavirus disease 2019 in China. N Engl J Med. 2020;382:1708-20.

24. Liu Z, Sun CB. Conjunctiva is not a preferred gateway of entry for SARS-CoV-2 to infect respiratory tract. J Med Virol. 2020;https:// doi.org/10.1002/jmv.25859.

25. Peng Y, Zhou YH. Is novel coronavirus disease (COVID-19) transmitted through conjunctiva? J Med Virol. 2020;https://doi.org/10. 1002/jmv.25753.

26. Guo D, Xia J, Shen Y, et al. SARS-CoV-2 may be related to conjunctivitis but not necessarily spread through the conjunctiva SARS-CoV-2 and conjunctiva. J Med Virol. 2020;https://doi.org/ 10.1002/jmv.25856.

27. Sun CB, Wang YY, Liu GH, Liu Z. Role of the eye in transmitting human coronavirus: what we know and what we do not know. Front Public Health. 2020;8:155.

28. Kuo IC. A Rashomon Moment? Ocular involvement and COVID19. Ophthalmology. 2020;127:984-5.

29. Chan WM, Yuen KS, Fan DS, Lam DS, Chan PK, Sung JJ. Tears and conjunctival scrapings for coronavirus in patients with SARS. Br J Ophthalmol. 2004;88:968-9.

30. Tong TR, Lam BH, Ng TK, Lai ST, Tong MK, Chau TN Conjunctiva-upper respiratory tract irrigation for early diagnosis of severe acute respiratory syndrome. J Clin Microbiol. 2003;41: 5352.

31. Wan Y, Shang J, Graham R, et al. Receptor recognition by the novel coronavirus from Wuhan: an analysis based on decade-long structural studies of SARS coronavirus. J Virol. 2020;94:e00127-0.

32. Ge XY, Li JL, Yang XL, Chmura AA, Zhu G, Epstein JH, et al. Isolation and characterization of a bat SARS-like coronavirus that uses the ACE2 receptor. Nature. 2013;503:535-8.

33. Seah I, Agrawal R. Can the coronavirus disease 2019 (COVID-19) affect the eyes? A review of coronaviruses and ocular implications in humans and animals. Ocul Immunol Inflamm. 2020;28:391-5. 
34. Sadhu S, Agrawal R, Pyare R, Pavesio C, Zierhut M, Khatri A, et al. COVID-19: limiting the risks for eye care professionals. Ocul Immunol Inflamm. 2020;28:714-20.

35. Lange C, Wolf J, Auw-Haedrich C, et al. Expression of the COVID-19 receptor ACE2 in the human conjunctiva. J Med Virol. 2020; https://doi.org/10.1002/jmv.25981.

36. Sun Y, Liu L, Pan X, et al. Mechanism of the action between the SARS-CoV S240 protein and the ACE2 receptor in eyes. Int J Ophthalmol (GUOJI YANKE ZAZHI). 2006;6:783-6.

37. Hui KPY, Cheung MC, Perera RAPM, Ng KC, Bui CHT, Ho JCW, et al. Tropism, replication competence, and innate immune responses of the coronavirus SARS-CoV-2 in human respiratory tract and conjunctiva: an analysis in ex-vivo and in-vitro cultures. Lancet Respir Med. 2020;8:687-95.

38. Hong N, Yu W, Xia J, et al. Evaluation of ocular symptoms and tropism of SARS-CoV-2 in patients confirmed with COVID-19. Acta Ophthalmol. 2020;https://doi.org/10.1111/aos.14445.

39. Belser JA, Rota PA, Tumpey TM. Ocular tropism of respiratory viruses. Microbiol Mol Biol Rev. 2013;77:144-56.

40. van der Hoek L, Pyrc K, Jebbink MF, Vermeulen-Oost W, Berkhout RJM, Wolthers KC, et al. Identification of a new human coronavirus. Nat Med. 2004;10:368-73.
41. Vabret A, Mourez T, Dina J, van der Hoek L, Gouarin S, Petitjean J, et al. Human coronavirus NL63, France. Emerg Infect Dis. 2005;11:1225-9.

42. Loon SC, Teoh SC, Oon LL, Se-Thoe SY, Ling AE, Leo YS, et al. The severe acute respiratory syndrome coronavirus in tears. Br J Ophthalmol. 2004;88:861-3.

43. Nowotny N, Kolodziejek J. Middle East respiratory syndrome coronavirus (MERS-CoV) in dromedary camels, Oman, 2013. Euro Surveill. 2014;19:20781.

44. Olivia Li JP, Shantha J, Wong TY, Wong EY, Mehta J, Lin H, et al. Preparedness among ophthalmologists: during and beyond the COVID-19 pandemic. Ophthalmology. 2020;127:569-72.

45. Britt JM, Clifton BC, Barnebey HS, Mills RP. Microaerosol formation in noncontact 'air-puff' tonometry. Arch Ophthalmol. 1991;109:225-8.

Publisher's Note Springer Nature remains neutral with regard to jurisdictional claims in published maps and institutional affiliations. 